\title{
Targeting mantle cell lymphoma metabolism and survival through simultaneous blockade of mTOR and nuclear transporter exportin-1
} This article has been corrected. Correction in: Oncotarget. 2019; 10:6842-6842.

Kazumasa Sekihara ${ }^{1,2}$, Kaori Saitoh ${ }^{1}$, Lina Han $^{3}$, Stefan Ciurea ${ }^{3}$, Shinichi Yamamoto ${ }^{1,2}$, Mika Kikkawa ${ }^{4}$, Saiko Kazuno ${ }^{4}$, Hikari Taka ${ }^{4}$, Naoko Kaga ${ }^{4}$, Hajime Arai $^{4}$, Takashi Miida ${ }^{1}$, Michael Andreeff ${ }^{3}$, Marina Konopleva ${ }^{3}$, Yoko Tabe ${ }^{1,3,5}$

${ }^{1}$ Department of Laboratory Medicine, Juntendo University Graduate School of Medicine, Tokyo, Japan

${ }^{2}$ Leading Center for the Development and Research of Cancer Medicine, Juntendo University Graduate School of Medicine, Tokyo, Japan

${ }^{3}$ Section of Molecular Hematology and Therapy, Department of Leukemia, The University of Texas MD Anderson Cancer Center, Houston, Texas, USA

${ }^{4}$ Laboratory of Proteomics and Biomolecular Science, Research Support Center, Juntendo University Graduate School of Medicine, Tokyo, Japan

${ }^{5}$ Department of Next Genertion Hematology Laboratory Medicine, Juntendo University Graduate School of Medicine, Tokyo, Japan Correspondence to: Yoko Tabe, email: tabe@juntendo.ac.jp Keywords: mantle cell lymphoma, mTORC1/2, selective inhibitor of nuclear export, XPO1, metabolism Received: May 31, $2016 \quad$ Accepted: March 16, $2017 \quad$ Published: March 27, 2017

Copyright: Sekihara et al. This is an open-access article distributed under the terms of the Creative Commons Attribution License (CC-BY), which permits unrestricted use, distribution, and reproduction in any medium, provided the original author and source are credited.

\section{ABSTRACT}

Mantle cell lymphoma (MCL) is an aggressive B-cell lymphoma with poor prognosis, characterized by aberrant expression of growth-regulating and oncogenic effectors and requiring novel anticancer strategies. The nuclear transporter exportin-1 (XPO1) is highly expressed in MCL and is associated with its pathogenesis. mTOR signaling, a central regulator of cell metabolism, is frequently activated in MCL and is also an important therapeutic target in this cancer. This study investigated the antitumor effects and molecular/metabolic changes induced by the combination of the small-molecule selective inhibitor XPO1 inhibitor KPT-185 and the dual mTORC1/2 kinase inhibitor AZD-2014 on MCL cells. AZD-2014 enhanced the KPT-185-induced inhibition of cell growth and repression of cell viability. The combination of KPT-185 and AZD-2014 downregulated c-Myc and heat shock factor 1 (HSF1) with its target heat shock protein 70 (HSP70). As a consequence, the combination caused repression of ribosomal biogenesis demonstrated by ITRAQ proteomic analyses. Metabolite assay by CETOF-MS showed that AZD-2014 enhanced the KPT-185-induced repression of MCL cellular energy metabolism through the TCA (Krebs) cycle, and further repressed KPT-185-caused upregulation of glycolysis.

Thus the simultaneous inhibition of XPO1 and mTOR signaling is a novel and promising strategy targeting prosurvival metabolism in MCL.

\section{INTRODUCTION}

Mantle cell lymphoma (MCL) is an aggressive B-cell lymphoma characterized by the aberrant expression of several growth-regulating, oncogenic effectors and is frequently resistant to standard chemotherapy [1]. Although MCL is characterized by the $\mathrm{t}(11,14)(\mathrm{q} 13 ; 32)$ translocation that results in aberrant expression of cyclin D1 [2], cyclin D1 overexpression itself is not sufficient for development of MCL; additional genetic events are necessary [3]. Exportin 1 (XPO1) mediates nuclear export of numerous molecules, including oncogenic transcription factors, ribosomal subunits, and RNAs, and is critical for cancer survival and proliferation. Elevated expression of XPO1 has been reported in the hematologic and solid tumors [4], and we have reported that the overexpression of XPO1 is associated with poor clinical outcomes in MCL and AML [5, 6]. Small-molecule selective inhibitors of nuclear export (SINE), which specifically and irreversibly bind to the Cys528 residue in the cargo-binding groove of XPO1, block XPO1-dependent nuclear export [4]. We and others demonstrated that the SINE compound KPT-185 [7] blocks XPO1 function and exerts an anti-lymphoma effect on MCL cells by suppression of oncogenic mediators such 
as cyclin D1; repression of ribosomal biogenesis, and downregulation of translation/chaperone proteins [5, 8, 9]. However, we found that XPO1 inhibition by KPT-185 resulted in upregulation of glycolysis [9], which plays an important role in sustaining tumor growth [10]. The result allows us to develop combination strategies with other molecular-targeted agents. Cancer cell proliferation and oncogenesis are coupled to metabolic reprogramming [11], such as PI3K/AKT signaling to directly stimulate glycolytic metabolism [11]. Cancer cells are known to consume glucose at a higher rate than their normal counterparts and produce lactate rather than completely oxidizing the glucose-derived carbon, a phenomenon known as the Warburg effect [12].

$\mathrm{PI} 3 \mathrm{~K} / \mathrm{AKT} / \mathrm{mTOR}$ signaling plays an important role in regulating MCL cell growth and proliferation [13], especially mTOR signaling, which is known to control protein synthesis by inducing ribosome biogenesis and mRNA translation [14] and is a critical pathway in the biology of MCL [15]. mTOR exists in two functionally and structurally distinct complexes, mTOR complex 1 (mTORC1) and mTORC2.The essential core component of mTORC1 is RAPTOR (regulatory-associated protein of mTOR) and that of mTORC2 is RICTOR (rapamycininsensitive companion of mTOR) [16]. Activated mTORC1 phosphorylates ribosomal S6 kinase (S6K), thereby activating it to subsequently phosphorylate ribosomal protein S6 and promote ribosome biogenesis, and further stimulates the metabolic pathways that ultimately drive cell growth [17]. The first-generation mTOR inhibitors, which selectively target mTORC1, have been disappointing in their efficacy and clinical activity [18] because of their inability to inhibit mTORC2, one of the potential key factors in rapamycin resistance. The selective targeting of mTORC1 by first-generation mTOR inhibitors results in cell survival and metabolic regulation via $\mathrm{S} 6 \mathrm{~K} 1$ and $\mathrm{TSC} 1 / 2$ activation, which are involved in a negative-feedback loop to regulate mTORC2 levels [19]. AZD-2014 [20] is a second-generation dual mTORC1/2 inhibitor currently being tested in clinical trials, including renal cancer [21] and advanced solid tumors [22]. AZD-2014 exhibits specific activity against mTORC1/2 and thereby efficiently blocks the AKT/ mTOR signal transduction pathway [18] without negativefeedback induction of mTORC2 [23]. In addition, ATP-competitive inhibitors such as AZD-2014 more efficienly target mTORC1 and block phosphorylation of rapamycin-resistant mTORC1 outputs such as 4EBP1 phosphorylation at T37/46 (4EBP1-T37/46) and capdependent translation [24].

We reasoned that a SINE and an mTOR inhibitor together might have complementary anticancer propertiesand thus offer a rational therapeutic benefit in MCL. We therefore hypothesized that a combination of the XPO1 inhibitor KPT-185 andselective mTORC1/2 dual inhibitor AZD-2014 would demonstrate synergistic inhibition of MCL cell growth by repressing prosurvival metabolism in this cancer.

\section{RESULTS}

\section{Synergistic anti-proliferative effects of KPT- 185+AZD-2014 in MCL cells}

We first examined the effect of the XPO1 inhibitor KPT-185 and the mTORC1/2 inhibitor AZD-2014 on the proliferation of MCL cells. Four MCL cell lines were treated with KPT-185, AZD-2014, or a KPT185+AZD-2014 combination for 48 hours. Both KPT-185 and AZD-2014 resulted in a dose-dependent reduction of cell viability as assessed by the CCK-8 assay (median inhibitory concentrations of KPT-185: $67 \mathrm{nM}$ for Jeko-1, $41 \mathrm{nM}$ for Z138, $684 \mathrm{nM}$ for JVM-2, and $147 \mathrm{nM}$ for MINO; of AZD-2014: $77 \mathrm{nM}$ for Jeko-1, $108 \mathrm{nM}$ for Z138, $179 \mathrm{nM}$ for JVM-2, and $133 \mathrm{nM}$ for MINO). The synergistic nature of the pharmacological interaction between KPT-185 and AZD-2014, which caused profound cell growth inhibition in all four cell lines, was shown by isobologram analysis (Table 1). Flow cytometric analysis of PI-stained cell nuclei showed that treatment with KPT185+AZD-2014 decreased the number of cells in S phase, with concomitant $\mathrm{G}_{0} / \mathrm{G}_{1}$ phase accumulation, and increased the number of cells in sub- $\mathrm{G}_{1}$ phase compared to controls in all cell lines (Figure 1). We performed the additional KPT-185 combination experiments utilizing different mTORC1/2 inhibitors:, AZD-8055, a close analog of AZD2014 [25] and a different chemotype MLN0128 [26], both of which demonstrated similar growth-inhibitory activity (Supplementary Figure 1). We further detected the profound cytotoxic effects of KPT-185 and AZD-2014 combination in MCL primary cells but not in CD34 positive cells from normal bone marrow samples (Figure 2).

\section{Inhibition of ribosomal biogenesis through inactivation of HSF1 by KPT-185+AZD-2014 in MCL cells}

To investigate alterations in biological processes induced by KPT-185 and AZD-2014, we employed a proteomic approach using iTRAQ to measure modulation of protein expression in response to treatment with KPT-185 and AZD-2014, either as monotherapy or in combination. A total of 1572, 2049, 2251, and 2375 unique proteins were identified in Jeko-1, Z138, JVM-2, and MINO cells, respectively, including 106, 142, 169, and 147 proteins whose expression level was significantly altered by KPT-185, AZD-2014, or KPT-185+AZD-2014.

To identify the alterations common to both KPT-185 and AZD-2014, proteins altered consistently in at least two of four tested cells were extracted. Expression of 32 proteins was consistently altered by KPT-185 (7 upregulated, 25 downregulated), 25 proteins by 
Table 1: Combination indices for KPT-185+AZD-2014 in MCL cell lines

\begin{tabular}{ccccc}
\hline KPT-185+AZD2014 & Jeko-1 & Z138 & JVM2 & MINO \\
\hline CI50 & 0.72 & 1.06 & 0.51 & 0.69 \\
CI75 & 0.64 & 0.91 & 0.42 & 0.58 \\
CI90 & 0.62 & 0.84 & 0.38 & 0.56 \\
CI average & 0.66 & 0.94 & 0.44 & 0.61 \\
\hline
\end{tabular}

CI: combination index.

A

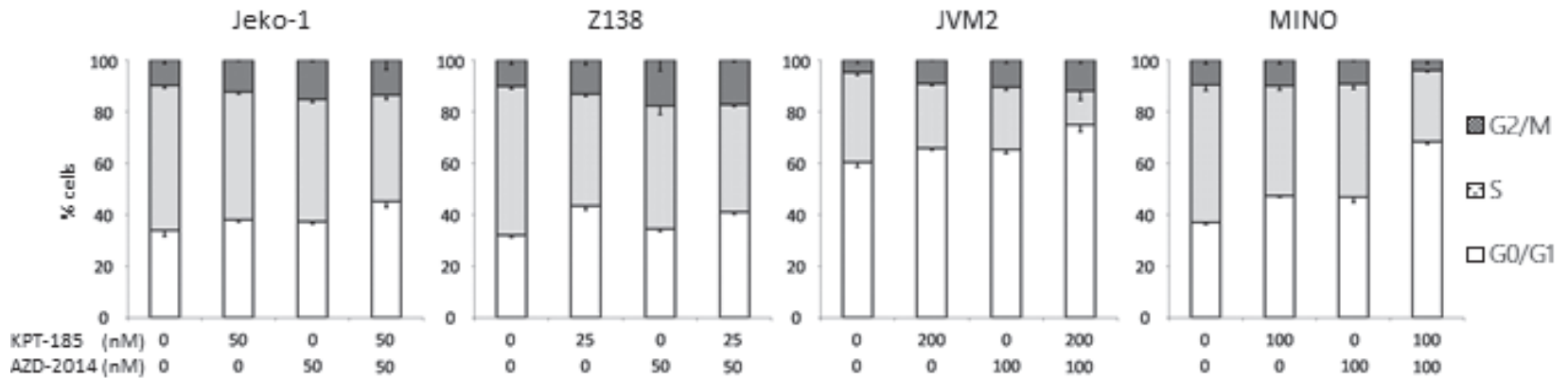

B

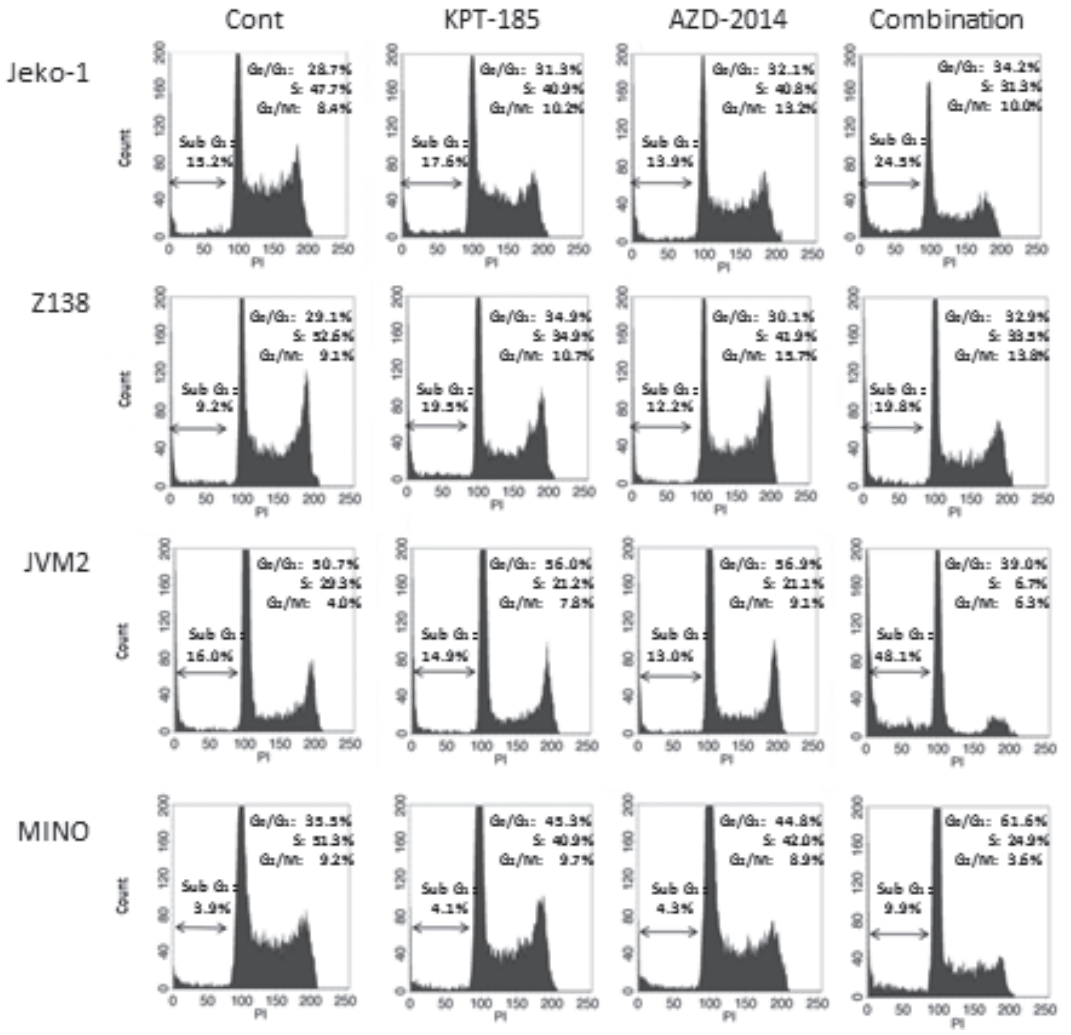

Figure 1: Induction of cell cycle arrest and apoptosis by the KPT-185 and AZD2014 combination in MCL cells. Jeko-1, Z138, JVM2, and MINO cells were treated by indicated concentrations of KPT-185, AZD-2014, or KPT-185+AZD-2014 (combination) for 72 hours (Jeko-1, Z138, and JVM2) or 48 hours (MINO). The DNA contents were measured by flow cytometry. Graphs show the means $\pm \mathrm{SD}$ of results of three independent experiments (A), and representative results show percentages of sub $\mathrm{G}_{1}$ cells (B). Cont; controls. 
AZD-2014 (10 upregulated, 15 downregulated), and 54 proteins by KPT-185+AZD-2014(25 upregulated, 29 downregulated) (Supplementary Table 2).

Notably, $68 \%$ of the proteins downregulated by KPT-185 (i.e., 17 of 25) were ribosomal proteins, suggesting that KPT-185 strongly inhibited ribosomal biogenesis, a finding consistent with our previous report [9]. KPT-185 repressed the importin protein, regulator of nucleocytoplasmic protein transport including ribosomal proteins [27], along with XPO1. AZD-2014 downregulated elongation factors (EF2, EF1A1) and heat shock chaperone proteins (HSP90B, HSP7C). The KPT185+AZD-2014 combination upregulated apoptosisassociated mitochondrial heat shock proteins and histone proteins.

We next examined the upstream regulators involved in the protein expression responses to KPT-185, AZD2014, or KPT-185+AZD-2014 in the MCL cells by IPA (Table 2). The IPA platform highlighted repression of the multifaceted transcription factor heat shock factor 1 (HSF1) by KPT-185+AZD-2014 and by AZD-2014 alone; HSF1 is known to be a central transducer linking the translational activity of ribosomal biogenesis and transcriptional regulation of heat shock proteins [28].
Downregulation of the oncogenic transcription factor Myc, E2F1, and mTOR target p70S6K also was highlighted after KPT-185+AZD-2014 treatment. IPA ontology analysis of cDNA array data for Jeko-1 cells demonstrated downregulation of NOD2 (nucleotidebinding oligomerization domain containing 2)(Table 3), a member of the Nod1/Apaf-1 family that is known to trigger activation of MAP kinases and of NF-kappa-B signaling [29]. Indeed, MAPK14 and NFKB1 were moderately but significantly inactivated by KPT185+AZD-2014 (z-score of IPA: MAPK14 -0.37, NFKB1 $-0.3, p<0.05)$. Whereas activation of tumor suppressor transcription factor TP53 has been observed after KPT185+AZD-2014 treatment (Table 2, Table 3), mutant TP53 bearing Jeko- 1 and MINO cells showed no change of TP53 expression level by immunoblotting (data not shown). These results suggest that anti-tumor effects of KPT-185+AZD-2014 combination are not dependent on TP53 status, consistent with previous reports $[8,9]$.

Immunoblot analysis demonstrated that AZD-2014 alone or combined with KPT-185 effectively suppressed p-S6 expression, and that the reduction of HSF1 phosphorylation and of c-Myc expression was caused by either KPT-185 and/or AZD-2014 in all MCL cells tested.

A
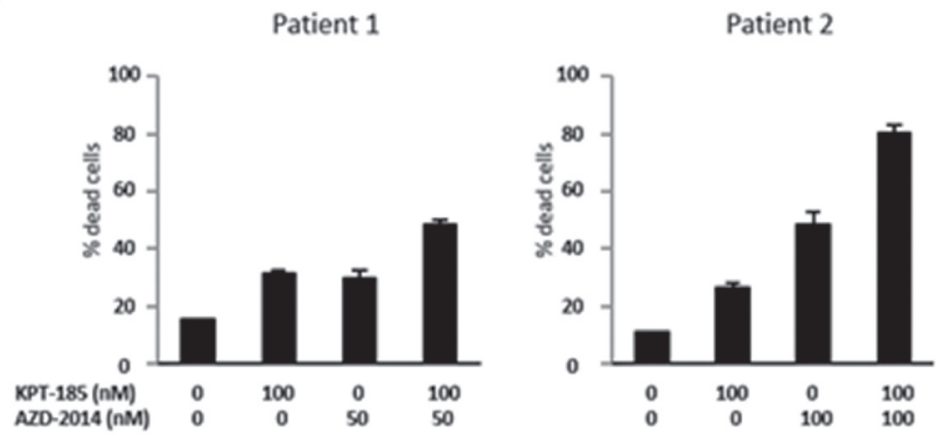

B

Sample 1

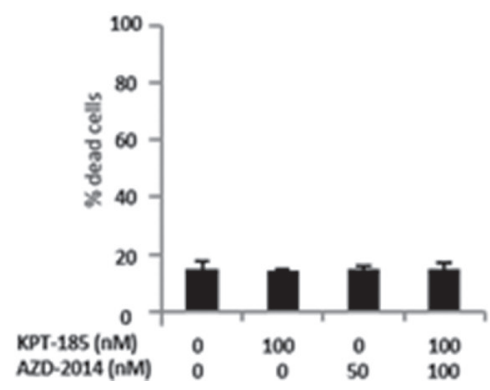

Sample 2

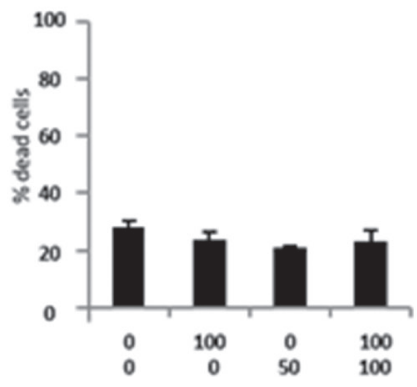

Sample 3

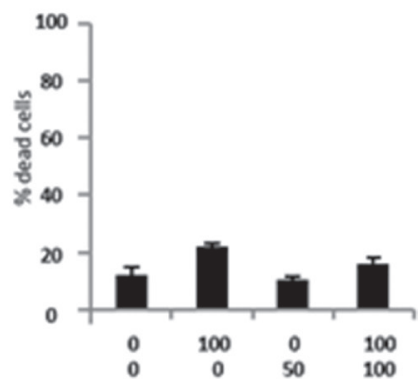

Figure 2: Cytotoxic effects of KPT-185 and AZD-2014 combination in primary MCL cells and not in normal hematopoietic stem cells. Cells were treated by indicated concentrations of KPT-185, AZD-2014, or KPT-185+AZD-2014 (combination) for 24 hours. (A) Primary MCL cells from two patients were assessed by the trypan blue exclusion cell count method. (B) The annexin $\mathrm{V}$ positivity was measured by flow cytometry after electronic gating on CD34+ stem/progenitor hematopoietic cells from normal bone marrow mononuclear cells from three normal donors as described in Materials and Methods. 
Table 2: Upstream factors involved in protein expression responses to KPT-185, AZD-2014, or KPT-185+AZD-2014in MCL cells

\begin{tabular}{|c|c|c|c|}
\hline Upstream Regulator & $\begin{array}{l}\text { Activation } \\
\text { z-score }\end{array}$ & $P$-value & Target molecules in dataset \\
\hline \multicolumn{4}{|l|}{ KPT-185 } \\
\hline \multicolumn{4}{|l|}{ Upregulated } \\
\hline RICTOR & 3.606 & $5.88 \mathrm{E}-18$ & $\begin{array}{l}P S M E 3, R P L 10, R P L 10 A, R P L 23, R P L 26, R P L 28, R P L 4, R P L 6, R P L 7 A, R P S 10, R P S 2 \text {, } \\
\text { RPS23, RPS3 }\end{array}$ \\
\hline TP53 & 1.432 & $8.79 \mathrm{E}-03$ & HMGB2, HSPA8, KPNA2, PSME3, VIM, XPO1 \\
\hline \multicolumn{4}{|l|}{ Downregulated } \\
\hline MYC & -3.719 & $9.94 \mathrm{E}-12$ & $\begin{array}{l}\text { EEF2, LGALS1, RPL10, RPL19, RPL23, RPL26, RPL27, RPL35, RPL5, RPL6, RPL7A, } \\
\text { RPS23, VIM, XPO1 }\end{array}$ \\
\hline MYCN & -3.499 & $2.88 \mathrm{E}-26$ & $\begin{array}{l}\text { EEF2, LGALS1, RPL10, RPL19, RPL23, RPL26, RPL27, RPL28, RPL35, RPL4, RPL5, } \\
\text { RPL6, RPS2, RPS23, RPS3, RPS4X, VIM }\end{array}$ \\
\hline HRAS & -1.982 & $6.54 \mathrm{E}-03$ & HSPA8, RPS10, RPS3, VIM \\
\hline \multicolumn{4}{|l|}{ AZD-2014 } \\
\hline \multicolumn{4}{|l|}{ Downregulated } \\
\hline HSF1 & -1.387 & $1.17 \mathrm{E}-06$ & CCT2, DNAJA1, HSP90AB1, HSPA8, HSPH1 \\
\hline \multicolumn{4}{|l|}{ KPT-185+AZD-2014 } \\
\hline \multicolumn{4}{|l|}{ Upregulated } \\
\hline $\mathrm{TNF}$ & 2.795 & $2.54 \mathrm{E}-03$ & FASN, HMGB1, HSP90AB1, HSPA8, HSPD1, PARP1, RRM1, RRM2, TPT1, VIM \\
\hline INSR & 2.224 & $1.10 \mathrm{E}-07$ & ACAA2, ATP5A1, CFL1, FASN, HSPD1, MDH2, PDCD4, RRM1, RRM2 \\
\hline TP53 & 2.109 & 2.67E-07 & $\begin{array}{l}\text { ACAA2, ADH5, FASN, HMGB1, HMGB2, HSP90AB1, HSPA8, HSPD1, KPNA2, MDH2, } \\
\text { PSME3, RRM1, RRM2, VIM, XPO1 }\end{array}$ \\
\hline PTEN & 1.724 & 5.33E-05 & ACAA2, FASN, G3BP1, PARP1, PDCD4, PGK1, RRM1 \\
\hline IFNG & 1.387 & $2.38 \mathrm{E}-02$ & EEF1A1, FASN, HMGB1, HSP90AB1, HSPA8, HSPD1, PSME3 \\
\hline OSM & 1.342 & $6.73 \mathrm{E}-03$ & ADH5, FASN, KRT9, PDCD4, PGK1 \\
\hline \multicolumn{4}{|l|}{ Downregulated } \\
\hline p70S6k & -1.982 & 1.03E-09 & EEF1A1, EEF2, FASN, PDCD4, VIM \\
\hline $\mathrm{E} 2 \mathrm{~F} 1$ & -1.673 & $1.21 \mathrm{E}-11$ & $\begin{array}{l}C C T 2, C S D E 1, H M G B 1, H M G B 2, H N R N P D, H S P A 8, H S P D 1, H S P E 1, K R T 1, N C L \text {, } \\
\text { RRM1, RRM2,VIM }\end{array}$ \\
\hline EGF & -1.627 & $1.10 \mathrm{E}-04$ & EEF1A1, FASN, NCL, PDCD4, RRM1, RRM2, VIM \\
\hline HSF1 & -1.458 & $2.45 \mathrm{E}-10$ & CCT2, DNAJA1, FASN, HMGB1, HSP90AB1, HSPA8, HSPD1, HSPE1, PGK1 \\
\hline MYCN & -1.387 & 7.63E-15 & $\begin{array}{l}\text { EEF1A1, EEF1G, EEF2, EIF4A1, HSP90AB1, HSPD1, NCL, RPL4, RPL6, RPS3A, RPS7, } \\
\text { TPI1, VIM }\end{array}$ \\
\hline
\end{tabular}

Data were analyzed by Ingenuity Pathway Analysis (IPA) based on the proteins whose expression was consistently altered after indicated treatment in Jeko-1, Z138, JVM2, and MINO cells (Supplementary Table 1).

(Figure 3A, Supplementary Figure 3A). Taken together, the XPO1 inhibition by KPT-185 and mTOR inhibition by AZD-2014 exhibited single-agent and/or combinatorial activities against the ribosomal biogenesis via inhibition of multiple factors including the transcription factor HSF1 and c-Myc.

\section{Inhibition of energy generation in MCL cells by KPT-185 and AZD-2014}

KEGG pathway analysis based on the iTRAQ proteomic data highlighted downregulation of glycolysis/ gluconeogenesis as the most significant pathway alteration 
Table 3: Upstream factors involved in transcriptional gene alterations by KPT-185, AZD-2014, or KPT-185+AZD-2014 in Jeko-1 cells

\begin{tabular}{lc}
\hline \multicolumn{1}{c}{ Upstream Regulator } & Activation z-score \\
\hline KPT-185+AZD-2014 & \\
Upregulated & \\
TP53 & $1.534 \pm 0.626$ \\
ESR1 & $1.959 \pm 0.118$ \\
$\quad$ Downregulated & \\
NOD2 & $-1.510 \pm 0.446$ \\
\hline
\end{tabular}

Data were analyzed by Ingenuity Pathway Analysis (IPA) based on the transcriptional genes that were consistently altered after indicated treatment inJeko-1 cells.

Values indicate the means \pm SEMs of activation z-scores in two independent experiments.

by AZD-2014 alone and by KPT-185+AZD-2014 $(p=0.035)$. We examined the metabolome profiling of MCL cells after KPT-185, AZD-2014, or KPT185+AZD-2014 treatment by CE-TOF-MS. A total of 93 and 56 metabolites were measured in Jeko-1 and Z138 cells, respectively (Supplementary Table 3). As expected from our previous findings [9], cells treated with KPT-185 showed higher levels of lactic acid than control cells. The KPT-185-induced upregulation of lactic acid was partially reversed by co-treatment with AZD-2014. We also observed decreases in levels of the tricarboxylic acid (TCA) or Krebs cycle metabolites, including citric acid, succinic acid, and malic acid, after treatment with single-agent KPT-185 or AZD-2014 Figure 4, decreases that were further abated by KPT185+AZD-2014. In order to examine whether AZD-2014 induced suppression of glycolysis, adaptively increased in response to KPT-185, promotes cell cycle arrest and apoptosis, we next conducted the experiments using the combination of glycolysis inhibitor 2DG [30, 31] and KPT-185. The combined treatment with KPT-185 and 2DG caused cell growth inhibition in all four cell lines (Supplementary Figure 2A). Notably, the combination of 2DG with KPT-185 exhibited the profound effects on cell cycle arrest and apoptosis induction with decreased the number of cells in $\mathrm{S}$ phase, concomitant $\mathrm{G} 0 / \mathrm{G} 1$ phase accumulation and accumulation of cells in sub-G1 phase, in the blastoid variant Z138 cells which is known to be highly proliferative and metabolically active [32-34], but only moderate to minimal effects in the classic typical MCL cells Jeko-1 [35], JVM-2 [34] and MINO [35]. (Supplementary Figure 2B).

Because KPT-185 and AZD-2014 combination suppressed multiple pathways of energy production including glycolysis and TCA cycle, we investigated whether activity of the energy stress marker AMPK is modulated by KPT-185 and/or AZD-2014. We examined the phosphorylation levels of AMPK $\alpha$ and of tuberous sclerosis complex 2 (TSC2), a substrate of AMPK [19], at 3 and 24 hours after treatment. AMPK $\alpha$ phosphorylation was moderately increased by AZD-2014 in all tested cells at different time points, and was not clearly stimulated affected upon combination with KPT185 (Figure 3B, Supplementary Figure 3C). On the other hand, KPT-185 and AZD-2014 combination increased TSC2 phosphorylation in Jeko-1 and MINO cells at 24 hour time-point. In JVM2 cells, AZD-2014 induced upregulation of phosphorylated TSC2 was not enhanced by KPT-185. Upregulation of phospho-TSC2 was observed in the blastoid variant Z138 by KPT-185 and/or AZD-2014 (Figure 3B, Supplementary Figure 3D). These results indicate that KPT-185 and AZD-2014 combination activates AMPK in a cell type-dependent manner.

\section{DISCUSSION}

The results presented here demonstrate that simultaneous inhibition of XPO1 by KPT-185 and mTORC1/2 kinase by AZD-2014 effectively decreased growth of MCL cells and inactivated the TCA cycle and glycolysis. We previously reported that single-agent KPT-185 exhibited anti-proliferative and pro-apoptotic activities in MCL cells by repressing ribosomal biogenesis as well as inhibiting nuclear export of transcription factors and oncogenic mRNAs [9]. Intriguingly, however, the KPT-185-treated MCL cells exhibited upregulation of glycolysis and gluconeogenesis pathways [9], which may negatively affect the drug's antitumor activity. We therefore assessed the efficacy of an inhibitor of mTOR signaling, a central regulator of cell metabolism integrating nutrients, combined with KPT-185 targeting the altered metabolism.

It has been reported that cancer tissues express higher levels of lactic acid, TCA metabolites, and amino acids than normal tissues, which may reflect a cancer-specific energy metabolism mechanism to secure the continuous proliferation of cancer cells in the limited resources of their microenvironment [36]. Amino acids funnel into the TCA cycle [37] is favored as a source of energy in cancer cells. mTOR signaling is known to stimulate specific metabolic pathways, including glycolysis, the pentose phosphate pathway, and lipid biosynthesis [38]. 
Importantly, our data suggest that the KPT185+AZD-2014 combination effectively inactivated energy metabolism of glycolysis and suppressed the
TCA cycle in MCL cells. Although KPT-185 decreased the levels of TCA cycle metabolites, it did stimulate the breakdown of pyruvate to lactate. mTOR inhibition by

A Jeko-1 $\mathrm{Z138}$ JVM-2

MINO

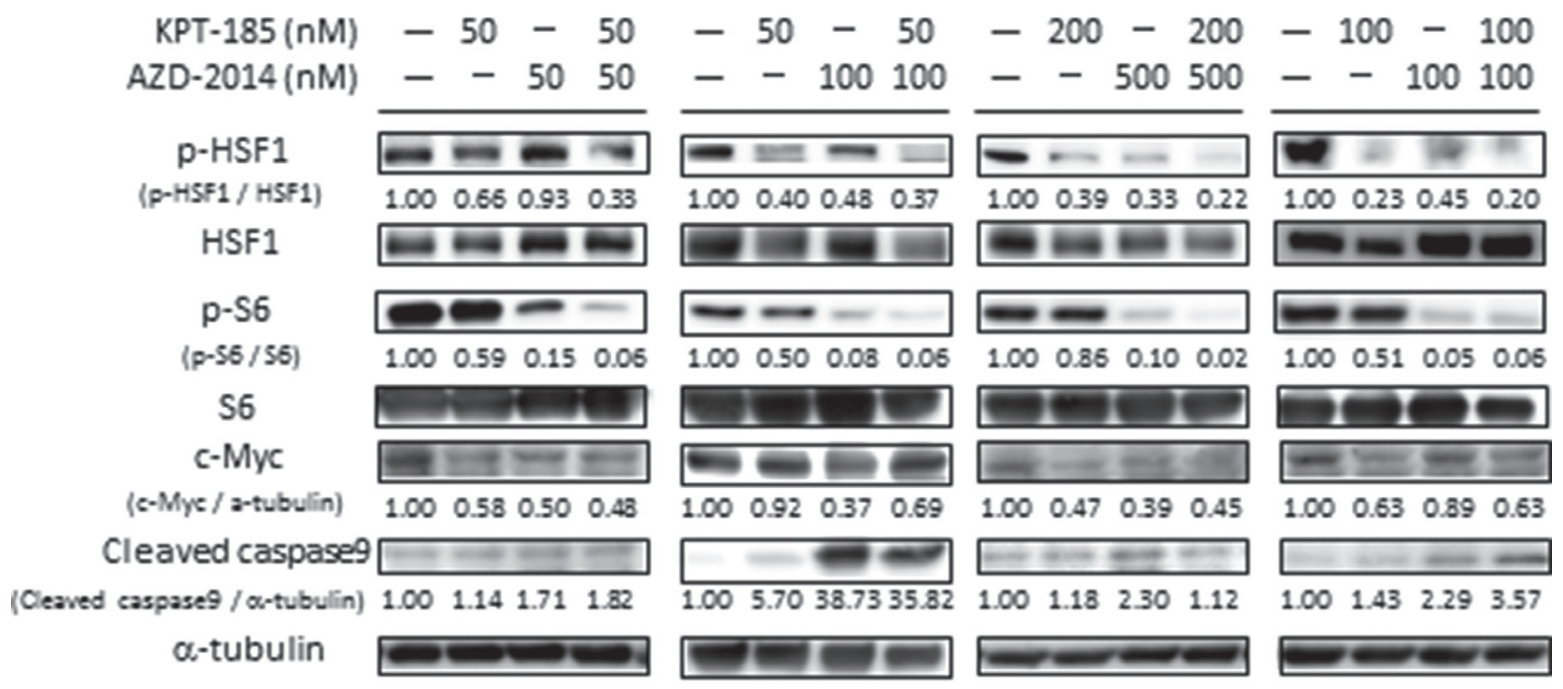

B

Jeko-1

$3 \mathrm{hr}$

\begin{tabular}{|c|c|c|c|c|c|c|c|c|}
\hline \multirow[b]{2}{*}{ KPT-185 (nM) } & \multicolumn{4}{|c|}{$3 \mathrm{hr}$} & \multicolumn{4}{|c|}{$24 \mathrm{hr}$} \\
\hline & - & 50 & - & 50 & - & 50 & - & 50 \\
\hline AZD-2014 (nM) & - & - & 50 & 50 & - & - & 50 & 50 \\
\hline p-AMPK $\alpha$ & 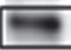 & 5 & 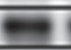 & 0 & $E$ & 5 & 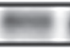 & $\theta$ \\
\hline AMPK $\alpha$ & $E$ & 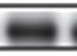 & 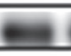 & $\theta$ & $E$ & $\theta$ & 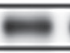 & $=$ \\
\hline (p-AMPK $\alpha / A M P K \alpha)$ & 1.00 & 1.18 & 1.76 & 2.37 & 1.00 & 1.15 & 1.09 & 1.90 \\
\hline $\mathrm{p}-\mathrm{TSC2}$ & 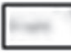 & 5 & - & $\cdots$ & 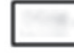 & +2 & $2=$ & - \\
\hline TSC2 & - & - & $=$ & - & $\square$ & 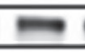 & 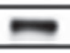 & $=$ \\
\hline$\left(p-\mathrm{TSC}_{2} / \mathrm{TSC} 2\right)$ & 1.00 & 5.71 & 4.99 & 5.91 & 1.00 & 3.52 & & 39.28 \\
\hline
\end{tabular}

JVM-2

$3 \mathrm{hr} \quad 24 \mathrm{hr}$

KPT-185(nM) - $200-200-200-200$

AZD-2014(nM) - - 500500 - 500500

p-AMPK $\alpha$

AMPK $\alpha$

(P-AMPKo / AMPK $\alpha$ )

p-TSC2

TSC2

(p-TSC2 / TSC2)

$\mathrm{Z} 138$

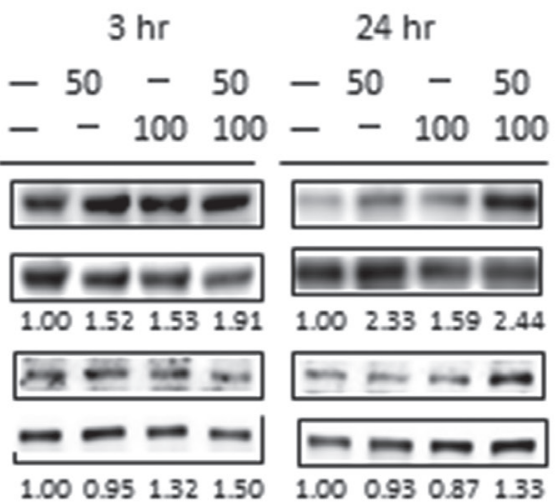

$$
\begin{gathered}
\text { MINO } \\
3 \mathrm{hr} 24 \mathrm{hr} \\
-100-100-100-100 \\
-\quad-100100--100100
\end{gathered}
$$

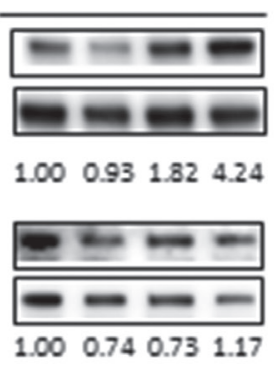

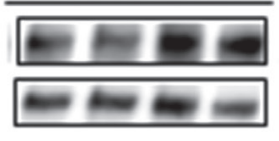

$1.00 \quad 0.78 \quad 1.46 \quad 2.26$

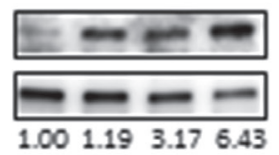

Figure 3: Molecular pathways affected by KPT-185 and AZD-2014 in MCL cells. After treatment for 24 hours (A), and 3 or 24 hours (B) with KPT-185, AZD-2014, or KPT-185+AZD-2014 (combination) at indicated concentrations, the cells indicated were subjected to lysis and immunoblot analysis. The results are representative of three independent experiments, and the intensity of each immunoblot signal compared to that of $\alpha$-tubulin was quantified using ImageJ software; the quantity is shown directly under each blot. 
AZD-2014 effectively reversed the KPT-185-induced activation of glycolysis, and promoted TCA cycle repression by KPT- 185 . We therefore propose that increased glycolysis might be an adaptive mechanism in response to KPT-185, which is, at least in part, suppressed by AZD-2014 to promote cell cycle arrest and death. The studies using glycolysis inhibitor 2DG combined with KPT-185 indeed demonstrated the cell growth inhibition in all examined cell lines with the most profound effects in the metabolically active blastoid variant MCL cells.

On the other hand, we observed that KPT-185 and AZD-2014 combination activated AMPK substrate TSC2 in the classic typical MCL cell lines but not in the blastoid variant MCL cells. The well-known clonal and genetic heterogeneity of MCL [2] could explain differential responses depending on MCL cell type seen in this study.

The antitumor effects of KPT-185 and AZD-2014 combination should be further confirmed in in vivo study. The multifaceted transcription factor HSF1, which was repressed by KPT-185+AZD-2014, coordinates net translational activity of ribosomal biogenesis and regulates a transcriptional network of genes driving heatshock proteins, protein synthesis, and energy metabolism as a prime transducer [28]. Taken together, our findings may have important implications for combining mTOR kinase inhibitors with XPO1 antagonists for the treatment of MCL (Figure 5). The anticancer effects we observed suggest a novel, rationally designed combinatorial strategy targeting prosurvival metabolism in MCL.

\section{MATERIALS AND METHODS}

\section{Cell cultures and reagents}

Four representative MCL cell lines, Jeko-1 [39], Z138 [40], JVM-2 [41], and MINO [42], were used in this study. Z138 cell line has a profile of the blastoid variant MCL [32, 34]; Jeko-1, JVM-2 and MINO have the classic typical phenotypes [34, 35]. Z138 and JVM2 cells have wt-TP53, and the Jeko-1 and MINO cells harbor mutant TP53 [35]. Cells were also treated with glycolysis inhibitor 2-Deoxy-D-glucose (2DG, Wako Pure Chemical Industries, Osaka, Japan) [30, 31], dual mTORC1/2 inhibitor AZD-8055 (LC laboratories, Woburn, MA) [25], MLN0128 (Selleck Chemicals, Houston, TX) [26]. Primary MCL samples and bone marrow mononuclear cells from healthy donors were obtained after informed consent in accordance with institutional guidelines set forth by Aichi Medical University and by MD Anderson Cancer Center per Declaration of Helsinki principles, respectively. MCL cell lines were cultured in RPMI 1640 medium containing 5\% fetal bovine serum and $1 \%$ penicillin/streptomycin, primary MCL cells were cultured in MEM $\alpha$ medium containing 10\% fetal bovine
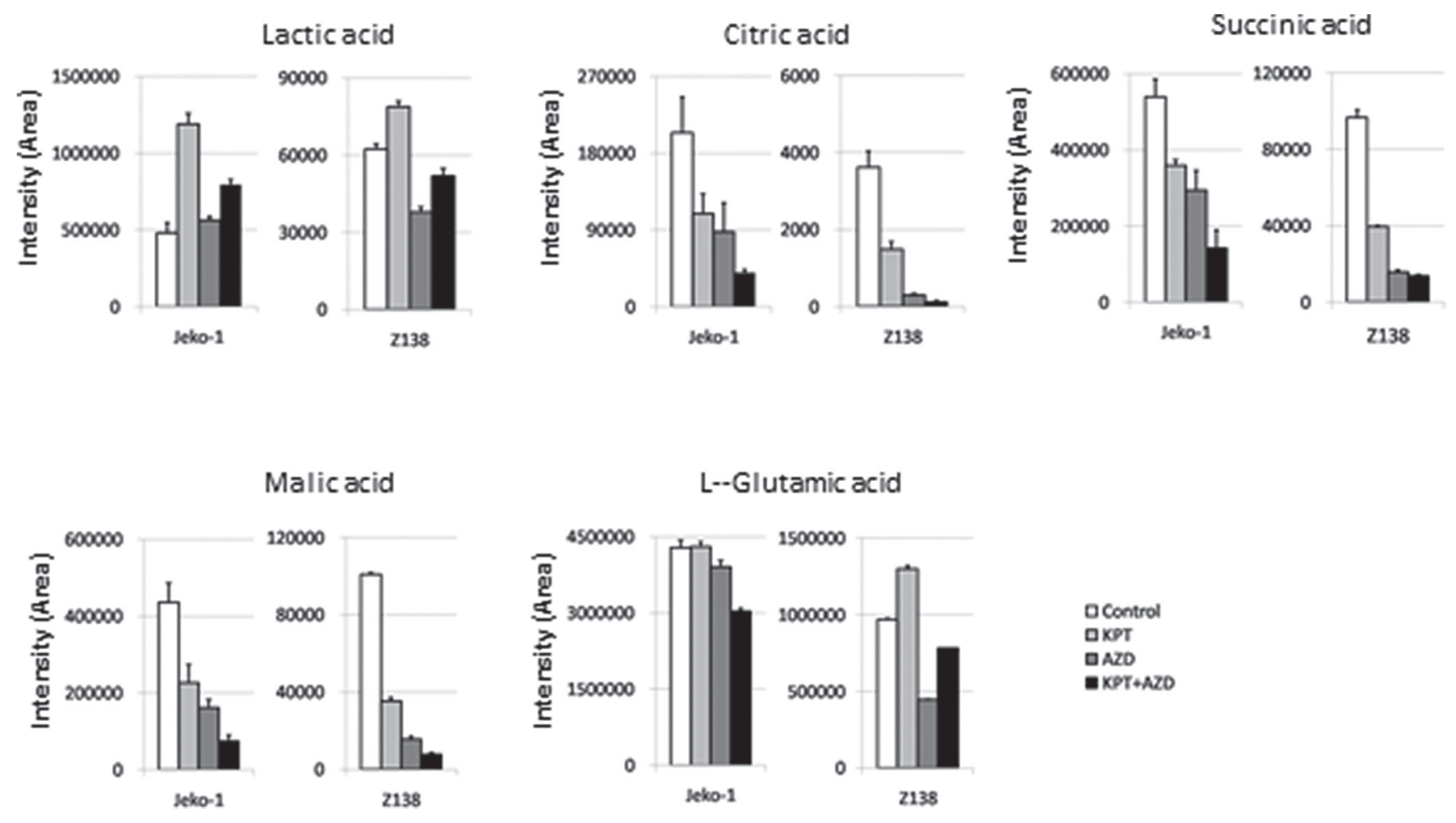

Figure 4: Quantification of metabolites affected by KPT-185, AZD-2014, or KPT-185+AZD-2014. The metabolites indicated were quantified inJeko-1 and Z138 cells treated with KPT-185, AZD-2014, or KPT-185+AZD-2014 (combination) for 18 hours (Jeko1:KPT-185 $50 \mathrm{nM}$, AZD-2014 $50 \mathrm{nM}$; Z138:KPT-185 $25 \mathrm{nM}$, AZD-2014 $50 \mathrm{nM}$ ) by CE-TOF-MS analysis. Graphs show the means $\pm \mathrm{SD}$ of results in two independent experiments. 
serum and $1 \%$ penicillin/streptomycin at $37^{\circ} \mathrm{C}$ in $5 \%$ $\mathrm{CO}_{2}$. Clinical characteristics of patients are summarized in Supplementary Table 1. Bone marrow mononuclear cells from healthy donors were seeded in StemSpan SFEM II (STEMCELL Technologies, Vancouver, BC, Canada), supplemented with BIT 9500 Serum Substitute (STEMCELL Technologies), cytokines including IL-3 (20 ng/ml), G-CSF (20 ng/ml), FLT3 ligand (50 ng/ml) and SCF (100 ng/ml) (all, Peprotech, Rocky Hill, NJ), StemReginin1 (Selleck Chemicals, Houston, TX) at $1 \mu \mathrm{M}$ and 10-4 $\beta$-Mercaptoethanol [43, 44]. Cells were treated with SINE compound KPT-185 (provided by Karyopharm Therapeutics Inc., Natick, MA) [7], dual mTORC1/2 inhibitor AZD-2014 (Selleck Chemicals, Houston, TX) [20], or both at the indicated concentrations.

\section{Cell growth, apoptosis, and cell-cycle analysis}

Proliferation of treated cells was determined by the CCK-8 colorimetric assay (Dojindo Molecular Technologies, Tokyo, Japan) according to the company's protocol. Apoptotic cell death and cell-cycle distribution in MCL cell lines were determined by flow cytometric analysis of propidium iodine (PI)-stained nuclei as described previously [45].

Cell death in primary MCL cells was assessed by the trypan blue exclusion cell count method [20]. Apoptosis in CD34+ stem/progenitor cells in normal bone marrow mononuclear cells was detected by flow cytometry using Annexin-V-APC (BD Biosciences, San Jose, CA), CD34FITC (BD Biosciences) and DAPI. Data were analyzed by using Flowjo software (Tree Star, Ashland, OR).

\section{iTRAQ sample labeling, mass spectrometry analysis, and peptide identification}

Proteins in treated cells were identified by isobaric tags for relative and absolute quantification (iTRAQ), a chemical labeling mass spectrometry (MS) method that was performed according to the manufacturer's protocol (AB SCIEX, Framingham, MA) [46, 47]. Briefly, the labeled peptides were analyzed by nano liquid chromatography in combination with tandem mass spectrometry (LC-MS/MS). Nano LC-MS was performed

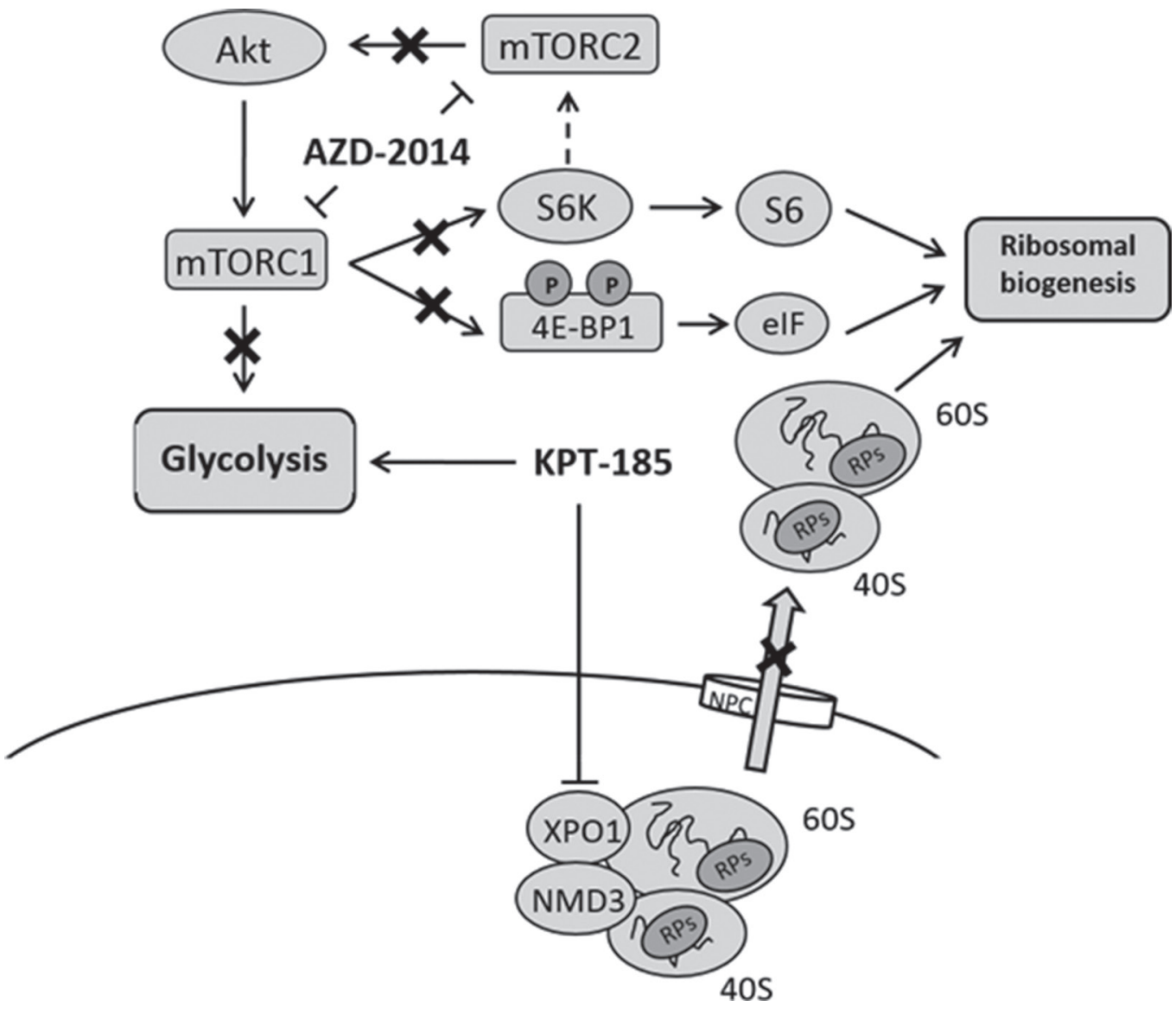

Figure 5: Combinational inhibition of XPO1 and mTOR impairs ribosomal biogenesis without glycolysis upregulation in MCL. KPT-185 inhibits XPO1 mediating nucleocytoplasmic export of ribosomal subunits and increases glycolysis. Dual mTORC1/2 kinase inhibitor AZD-2014 represses S6K activation and 4EBP1 phosphorylation without negative-feedback induction of mTORC2, which results in decrease of ribosome biogenesis. mTOR inhibition by AZD-2014 suppresses glycolysis. 
on nano LC system (AB SCIEX) using a ChromXP C18-CL column (Eksigent parts of AB SCIEX, Dublin, California, USA) and TripleTOF 5600 mass spectrometer for MS/MS (AB SCIEX) with Analyst TF 1.7 software. Protein identification and relative quantification were carried out by ProteinPilot Software Version 5.0 (AB SCIEX) [48]. The proteins identified were functionally defined by searching the UniProt database (Release 01/20/2016). Protein ratios were normalized against the overall median ratio for all the peptides in the sample for each separate ratio in every individual experiment. A confidence cutoff for protein identification of $>95 \%$ was applied. Proteins whose expression was statistically significantly changed by treatment were subjected to functional analysis by KEGG pathway enrichment analysis using DAVID Bioinformatics Resources [49] or the Ingenuity Pathway Analysis software (IPA,Ingenuity Systems, QIAGEN; www.qiagen.com/ingenuity) [9]. For IPA analysis, we used two scores: an 'enrichment' score (Fisher exact test $P$-value) that measures overlap of observed and predicted regulated gene sets and a Z-score that assesses the match of observed and predicted upregulation/downregulation patterns [50].

\section{Immunoblot analysis}

Cells were solubilized in lysis buffer comprising phosphate-buffered saline solution containing $1 \times$ cell lysis buffer (Cell Signaling Technology, Danvers, MA), 1× protease inhibitor cocktail (Roche, Indianapolis, IN), and $1 \times$ phosphatase inhibitor cocktail (Roche) and incubated for $30 \mathrm{~min}$ on ice. The lysates were then subjected to centrifugation for $10 \mathrm{~min}$ at $13,000 \mathrm{rpm}$ at $4^{\circ} \mathrm{C}$. Total protein concentrations were determined by the Bio-Rad Protein Assay Kit (Bio-Rad Laboratories, Hercules, CA) according to the manufacturer's instructions. Total proteins (40 $\mu \mathrm{g})$ were separated by sodium dodecyl sulfatepolyacrylamide gel electrophoresis (Bio-Rad Laboratories) and transferred to polyvinylidene-fluoride membranes ( $0.45 \mu \mathrm{m}$, Millipore, Bedford, MA), then probed with first and second antibodies according to the manufacturers' protocols. The following antibodies were used: $\alpha$-tubulin (Sigma-Aldrich, St Louis, MO), p-HSF1 ${ }^{\text {Ser326 }}$ (Abcam, Cambridge, MA), HSF1, p-S6 ribosomal protein (S6K) Ser235/Ser236, S6 ribosomal protein, c-Myc, cleaved caspase-9, p-AMPKa ${ }^{\text {Thr172, }}$ AMPK $\alpha$, p-TSC2 $^{\text {Thr1462 }}$, TSC2 and horseradish peroxidase-linked anti-mouse and anti-rabbit IgG (all, Cell Signaling Technology).

\section{Metabolite measurements}

Metabolic extracts were prepared from cells $\left(2-5 \times 10^{6}\right)$ by the HMT method (Human Metabolome Technologies, Inc., Tsuruoka, Japan) and analyzed on a capillary electrophoresis (CE)-quadruple-time of flight (Q TOF ) MS system (7100 capillary electrophoresis
- 6530 Accurate Q TOF Mass; Agilent Technologies Inc. Santa Clara, CA). Briefly, the culture medium was removed from each dish, and the cells were washed twice in $5 \%$ mannitol solution $(10 \mathrm{~mL}$, then $2 \mathrm{~mL}) .1000 \mu \mathrm{L}$ of methanol containing internal standard (H3304-1002, Human Metabolome Technologies, Inc. Santa Clara, CA), $1000 \mu \mathrm{L}$ of Chloroform and $400 \mu \mathrm{L}$ of Milli-Q water were added to the cells and then centrifuged at $2,300 \times \mathrm{g}$ at $4^{\circ} \mathrm{C}$ for 5 minutes. Metabolite solutions were filtrated by Millipore 5-kDa cutoff filter at $9,100 \times \mathrm{g}$ at $4^{\circ} \mathrm{C}$ for 120 minutes and dried. The metabolites were resuspended in $50 \mu \mathrm{L}$ of Milli-Q water containing internal standard (H3304-1004, Human Metabolome Technologies, Inc.) and applied to CE-MS. CE-MS experiments were carried out in positive and negative mode according to the methods developed by Soga et al $[51,52]$. The obtained metabolite peaks were analyzed using Mass Profiler Professional (MPP) software (Agilent Technologies, Inc. Santa Clara, CA.)

\section{cDNA microarray}

Gene expression in the cells was determined by microarray analysis using the Affymetrix Human Gene 2.0 ST Array according to Affymetrix protocols (Santa Clara, CA). Signal intensities were measured by using a GeneChip Scanner3000 7G (Affymetrix) and converted to numerical data by using the Affymetrix Expression Console Software 1.3.1 (Affymetrix). To identify candidate genes of potential significance in MCL, we applied a 1.5 -fold change cutoff, since the combined responses of a group of genes acting in concert might affect the physiology of the cell, as previously described [53]. The digitized data were analyzed by GeneSpring GX 13.1.0 software (Agilent Technologies, Santa Clara, CA, USA).

\section{Statistical analyses}

Groups were compared by a two-tailed Student $t$-test. A $P$-value $\leq 0.05$ was considered statistically significant. Where indicated, the results are expressed as the mean \pm standard deviation (SD) of triplicate samples. Synergism, additive effects, and antagonism were assessed by the Chou-Talalay method [54], utilizing Calcusyn software (Biosoft, Cambridge, UK). The effect on cellular proliferation was shown as a percentage reduction of cell viability when compared with dimethyl sulfoxide-treated controls. The average combination index (CI) value for the experimental combination was calculated from the $50 \%, 75 \%$, and $90 \%$ effective doses (ED50, ED75, and ED90). By this method, CI values indicate the following: $0.3-0.7$, strong synergism; $0.7-$ 0.85 , moderate synergism; 0.85-0.9, slight synergism; 0.9-1.1, nearly additive; 1.1-1.2, slight antagonism; 1.2-1.45, moderate antagonism; 1.45-3.3, antagonism; 3.3-10, strong antagonism [54]. 


\section{Authors' contributions}

Conception and design: K. Sekihara, Y. Tabe, M. Andreeff, and M. Konopleva; development of methodology: K. Sekihara, L. Han, S. Ciurea and Y. Tabe; acquisition of data: K. Sekihara, Y. Tabe, S. Yamamoto, K. Saitoh, M. Kikkawa, S. Kazuno, H. Taka, and N. Kaga; analysis and interpretation of data: K. Sekihara, Y. Tabe, and $\mathrm{H}$. Matsushita; writing, review, and/or revision of the manuscript: K. Sekihara, Y. Tabe, M. Andreeff, and M. Konopleva; administrative, technical, or material support: T. Miida; study supervision: M. Konopleva.

\section{ACKNOWLEDGMENTS}

The authors wish to thank Dr. Hideki Hayashi and Dr. Masayuki Tanaka, Education and Research Support Center, Tokai University, for statistical support and Dr. Numsen Hail, Jr., for his help in preparation of the manuscript. We thank the Divisions of Molecular and Biochemical Research and Cell Biology, Juntendo University Graduate School of Medicine, for use of facilities. This work was supported in part by a Grant-inAid for Young Scientists (B) (16K19855) from the MEXT, Japan (to K.S.), by a Grant-in-Aid for Scientific Research (C)(15K08653) and by a Grant-in-Aid (S1311011) from the Foundation for Strategic Research Projects in Private Universities from the MEXT, Japan (to Y.T.), by U.S. National Institutes of Health awards (CA055164, CA083639, CA136411, CA100632),by the MD Anderson Cancer Center Support Grant (CA016672), and by the Paul and Mary Haas Chair in Genetics (to M.A.).

We thank Kathryn Hale for manuscript review and Melodie England for help in the preparation of the manuscript.

\section{CONFLICTS OF INTEREST}

The authors have no conflicts of interest to declare.

\section{REFERENCES}

1. Martin P, Chadburn A, Christos P, Furman R, Ruan J, Joyce MA, Fusco E, Glynn P, Elstrom R, Niesvizky R, Feldman EJ, Shore TB, Schuster MW, et al. Intensive treatment strategies may not provide superior outcomes in mantle cell lymphoma: overall survival exceeding 7 years with standard therapies. Ann Oncol. 2008; 19:1327-30.

2. Perez-Galan P, Dreyling M, Wiestner A. Mantle cell lymphoma: biology, pathogenesis, and the molecular basis of treatment in the genomic era. Blood. 2011; 117:26-38.

3. Bernard M, Gressin R, Lefrere F, Drenou B, Branger B, Caulet-Maugendre S, Tass P, Brousse N, Valensi F, Milpied N, Voilat L, Sadoun A, Ghandour C, et al.
Blastic variant of mantle cell lymphoma: a rare but highly aggressive subtype. Leukemia. 2001; 15:1785-91.

4. Etchin J, Sun Q, Kentsis A, Farmer A, Zhang ZC, Sanda T, Mansour MR, Barcelo C, McCauley D, Kauffman M, Shacham S, Christie AL, Kung AL, et al. Antileukemic activity of nuclear export inhibitors that spare normal hematopoietic cells. Leukemia. 2013; 27:66-74.

5. Yoshimura M, Ishizawa J, Ruvolo V, Dilip A, QuintasCardama A, McDonnell TJ, Neelapu SS, Kwak LW, Shacham S, Kauffman M, Tabe Y, Yokoo M, Kimura S, et al. Induction of p53-mediated transcription and apoptosis by exportin-1 (XPO1) inhibition in mantle cell lymphoma. Cancer Sci. 2014; 105:795-801.

6. Kojima K, Kornblau SM, Ruvolo V, Dilip A, Duvvuri S, Davis RE, Zhang M, Wang Z, Coombes KR, Zhang N, Qiu YH, Burks JK, Kantarjian H, et al. Prognostic impact and targeting of CRM1 in acute myeloid leukemia. Blood. 2013; 121:4166-74.

7. Ranganathan $\mathrm{P}, \mathrm{Yu} \mathrm{X}, \mathrm{Na} \mathrm{C}$, Santhanam R, Shacham S, Kauffman M, Walker A, Klisovic R, Blum W, Caligiuri M, Croce CM, Marcucci G, Garzon R. Preclinical activity of a novel CRM1 inhibitor in acute myeloid leukemia. Blood. 2012; 120:1765-73.

8. Zhang K, Wang M, Tamayo AT, Shacham S, Kauffman M, Lee J, Zhang L, Ou Z, Li C, Sun L, Ford RJ, Pham LV. Novel selective inhibitors of nuclear export CRM1 antagonists for therapy in mantle cell lymphoma. Experimental hematology. 2013; 41:67-78. e4.

9. Tabe Y, Kojima K, Yamamoto S, Sekihara K, Matsushita H, Davis RE, Wang Z, Ma W, Ishizawa J, Kazuno S, Kauffman M, Shacham S, Fujimura T, et al. Ribosomal Biogenesis and Translational Flux Inhibition by the Selective Inhibitor of Nuclear Export (SINE) XPO1 Antagonist KPT-185. PLoS One. 2015; 10:e0137210.

10. Fantin VR, St-Pierre J, Leder P. Attenuation of LDH-A expression uncovers a link between glycolysis, mitochondrial physiology, and tumor maintenance. Cancer Cell. 2006; 9:425-34.

11. Ward PS, Thompson CB. Metabolic reprogramming: a cancer hallmark even warburg did not anticipate. Cancer Cell. 2012; 21:297-308.

12. Vander Heiden MG, Cantley LC, Thompson CB. Understanding the Warburg effect: the metabolic requirements of cell proliferation. Science. 2009; 324:1029-33.

13. Tabe Y, Jin L, Konopleva M, Shikami M, Kimura S, Andreeff M, Raffeld M, Miida T. Class IA PI3K inhibition inhibits cell growth and proliferation in mantle cell lymphoma. Acta Haematol. 2014; 131:59-69.

14. Ma XM, Blenis J. Molecular mechanisms of mTORmediated translational control. Nat Rev Mol Cell Biol. 2009; 10:307-18.

15. Blachly JS, Baiocchi RA. Targeting PI3-kinase (PI3K), AKT and mTOR axis in lymphoma. Br J Haematol. 2014; 167:19-32. 
16. Laplante M, Sabatini DM. mTOR signaling in growth control and disease. Cell. 2012; 149:274-93.

17. Shimobayashi M, Hall MN. Making new contacts: the mTOR network in metabolism and signalling crosstalk. Nat Rev Mol Cell Biol. 2014; 15:155-62.

18. Eyre TA, Collins GP, Goldstone AH, Cwynarski K. Time now to TORC the TORC? New developments in mTOR pathway inhibition in lymphoid malignancies. $\mathrm{Br} \mathrm{J}$ Haematol. 2014; 166:336-51.

19. Huang J, Manning BD. The TSC1-TSC2 complex: a molecular switchboard controlling cell growth. Biochem J. 2008; 412:179-90.

20. Harada M, Benito J, Yamamoto S, Kaur S, Arslan D, Ramirez S, Jacamo R, Platanias L, Matsushita H, Fujimura T, Kazuno S, Kojima K, Tabe Y, et al. The novel combination of dual mTOR inhibitor AZD2014 and pan-PIM inhibitor AZD1208 inhibits growth in acute myeloid leukemia via HSF pathway suppression. Oncotarget. 2015; 6:37930-47. doi: 10.18632/oncotarget.6122.

21. Powles $\mathrm{T}$, Wheater $\mathrm{M}$, Din O, Geldart T, Boleti E, Stockdale A, Sundar S, Robinson A, Ahmed I, Wimalasingham A, Burke W, Sarker SJ, Hussain S, et al. A Randomised Phase 2 Study of AZD2014 Versus Everolimus in Patients with VEGF-Refractory Metastatic Clear Cell Renal Cancer. Eur Urol. 2016; 69:450-6.

22. Basu B, Dean E, Puglisi M, Greystoke A, Ong M, Burke W, Cavallin M, Bigley G, Womack C, Harrington EA, Green S, Oelmann E, de Bono JS, et al. First-in-Human Pharmacokinetic and Pharmacodynamic Study of the Dual m-TORC 1/2 Inhibitor AZD2014. Clin Cancer Res. 2015; 21:3412-9.

23. Rastogi R, Jiang Z, Ahmad N, Rosati R, Liu Y, Beuret L, Monks R, Charron J, Birnbaum MJ, Samavati L. Rapamycin induces mitogen-activated protein (MAP) kinase phosphatase-1 (MKP-1) expression through activation of protein kinase $\mathrm{B}$ and mitogen-activated protein kinase kinase pathways. J Biol Chem. 2013; 288:33966-77.

24. Thoreen CC, Kang SA, Chang JW, Liu Q, Zhang J, Gao Y, Reichling LJ, Sim T, Sabatini DM, Gray NS. An ATPcompetitive mammalian target of rapamycin inhibitor reveals rapamycin-resistant functions of mTORC1. J Biol Chem. 2009; 284:8023-32.

25. Willems L, Chapuis N, Puissant A, Maciel TT, Green AS, Jacque N, Vignon C, Park S, Guichard S, Herault O, Fricot A, Hermine O, Moura IC, et al. The dual mTORC1 and $\mathrm{mTORC} 2$ inhibitor AZD8055 has anti-tumor activity in acute myeloid leukemia. Leukemia. 2012; 26:1195-202.

26. Zeng Z, Wang RY, Qiu YH, Mak DH, Coombes K, Yoo SY, Zhang Q, Jessen K, Liu Y, Rommel C, Fruman DA, Kantarjian HM, Kornblau SM, et al. MLN0128, a novel mTOR kinase inhibitor, disrupts survival signaling and triggers apoptosis in AML and AML stem/ progenitor cells. Oncotarget. 2016; 7:55083-55097. doi: 10.18632/ oncotarget.10397.

27. Lott $\mathrm{K}$, Cingolani $\mathrm{G}$. The importin $\beta$ binding domain as a master regulator of nucleocytoplasmic transport. Biochim Biophys Acta. 2011; 1813:1578-92.
28. Santagata S, Mendillo ML, Tang YC, Subramanian A, Perley CC, Roche SP, Wong B, Narayan R, Kwon H, Koeva M, Amon A, Golub TR, Porco JA Jr, et al. Tight coordination of protein translation and HSF1 activation supports the anabolic malignant state. Science. 2013; 341:1238303.25.

29. Inohara N, Nuñez G. NODs: intracellular proteins involved in inflammation and apoptosis. Nat Rev Immunol. 2003; 3:371-82.

30. Halicka HD, Ardelt B, Li X, Melamed MM, Darzynkiewicz Z. 2-Deoxy-D-glucose enhances sensitivity of human histiocytic lymphoma U937 cells to apoptosis induced by tumor necrosis factor. Cancer Res. 1995; 55:444-9.

31. Pradelli LA, Bénéteau M, Chauvin C, Jacquin MA, Marchetti S, Muñoz-Pinedo C, Pende M, Ricci JE. Glycolysis inhibition sensitizes tumor cells to death receptors-induced apoptosis by AMP kinase activation leading to Mcl-1 block in translation. Oncogene. 2010; 29:1641-52.

32. Medeiros LJ, Estrov Z, Rassidakis GZ. Z-138 cell line was derived from a patient with blastoid variant mantle cell lymphoma. Leuk Res. 2006; 30:497-501.

33. Bernard M, Gressin R, Lefrère F, Drénou B, Branger B, Caulet-Maugendre S, Tass P, Brousse N, Valensi F, Milpied N, Voilat L, Sadoun A, Ghandour C, et al. Blastic variant of mantle cell lymphoma: a rare but highly aggressive subtype. Leukemia. 2001; 15:1785-91.

34. Tucker CA, Bebb G, Klasa RJ, Chhanabhai M, Lestou V, Horsman DE, Gascoyne RD, Wiestner A, Masin D, Bally M, Williams ME. Four human t(11;14)(q13;q32)containing cell lines having classic and variant features of Mantle Cell Lymphoma. Leuk Res. 2006; 30:449-57.

35. Amin HM, McDonnell TJ, Medeiros LJ, Rassidakis GZ, Leventaki V, O'Connor SL, Keating MJ, Lai R. Characterization of 4 mantle cell lymphoma cell lines. Arch Pathol Lab Med. 2003; 127:424-31.

36. Hirayama A, Kami K, Sugimoto M, Sugawara M, Toki N, Onozuka H, Kinoshita T, Saito N, Ochiai A, Tomita M, Esumi H, Soga T. Quantitative metabolome profiling of colon and stomach cancer microenvironment by capillary electrophoresis time-of-flight mass spectrometry. Cancer Res. 2009; 69:4918-25.

37. Argilés JM, Azcón-Bieto J. The metabolic environment of cancer. Mol Cell Biochem. 1988; 81:3-17.27.

38. Guertin DA, Sabatini DM. Defining the role of mTOR in cancer. Cancer Cell. 2007; 12:9-22.

39. Jeon HJ, Kim CW, Yoshino T, Akagi T. Establishment and characterization of a mantle cell lymphoma cell line. Br J Haematol. 1998; 102:1323-6.

40. Estrov Z, Talpaz M, Ku S, Harris D, Van Q, Beran M, Hirsch-Ginsberg C, Huh Y, Yee G, Kurzrock R. Z-138: a new mature B-cell acute lymphoblastic leukemia cell line from a patient with transformed chronic lymphocytic leukemia. Leuk Res. 1998; 22:341-53. 
41. Melo JV, Brito-Babapulle V, Foroni L, Robinson DS, Luzzatto L, Catovsky D. Two new cell lines from B-prolymphocytic leukaemia: characterization by morphology, immunological markers, karyotype and Ig gene rearrangement. Int J Cancer. 1986; 38:531-8.

42. Lai R, McDonnell TJ, O'Connor SL, Medeiros LJ, Oudat R, Keating M, Morgan MB, Curiel TJ, Ford RJ. Establishment and characterization of a new mantle cell lymphoma cell line, Mino. Leuk Res. 2002; 26:849-55.

43. Pabst C, Krosl J, Fares I, Boucher G, Ruel R, Marinier A, Lemieux S, Hébert J, Sauvageau G. Identification of small molecules that support human leukemia stem cell activity ex vivo. Nat Methods. 2014; 11:436-42.

44. Boitano AE, Wang J, Romeo R, Bouchez LC, Parker AE, Sutton SE, Walker JR, Flaveny CA, Perdew GH, Denison MS, Schultz PG, Cooke MP. Aryl hydrocarbon receptor antagonists promote the expansion of human hematopoietic stem cells. Science. 2010; 329:1345-8.

45. Milella M, Kornblau SM, Estrov Z, Carter BZ, Lapillonne H, Harris D, Konopleva M, Zhao S, Estey E, Andreeff M. Therapeutic targeting of the MEK/MAPK signal transduction module in acute myeloid leukemia. J Clin Invest. 2001; 108:851-9.

46. Glen A, Gan CS, Hamdy FC, Eaton CL, Cross SS, Catto JW, Wright PC, Rehman I. iTRAQ-facilitated proteomic analysis of human prostate cancer cells identifies proteins associated with progression. J Proteome Res. 2008; 7:897-907.

47. Herbrich SM, Cole RN, West KP, Schulze K, Yager JD, Groopman JD, Christian P, Wu L, O'Meally RN, May DH, McIntosh MW, Ruczinski I. Statistical inference from multiple iTRAQ experiments without using common reference standards. J Proteome Res. 2013; 12:594-604.
48. Longworth J, Noirel J, Pandhal J, Wright PC, Vaidyanathan S. HILIC- and SCX-based quantitative proteomics of Chlamydomonas reinhardtii during nitrogen starvation induced lipid and carbohydrate accumulation. J Proteome Res. 2012; 11:5959-71.

49. Ekins S, Bugrim A, Brovold L, Kirillov E, Nikolsky Y, Rakhmatulin E, Sorokina S, Ryabov A, Serebryiskaya T, Melnikov A, Metz J, Nikolskaya T. Algorithms for network analysis in systems-ADME/Tox using the MetaCore and MetaDrug platforms. Xenobiotica. 2006; 36:877-901.

50. Krämer A, Green J, Pollard J, Tugendreich S. Causal analysis approaches in Ingenuity Pathway Analysis. Bioinformatics. 2014; 30:523-30.

51. Soga $T$, Ohashi $Y$, Ueno $Y$, Naraoka $H$, Tomita $M$, Nishioka T. Quantitative metabolome analysis using capillary electrophoresis mass spectrometry. J Proteome Res. 2003; 2:488-94.

52. Kami K, Fujimori T, Sato H, Sato M, Yamamoto H, Ohashi Y, Sugiyama N, Ishihama Y, Onozuka H, Ochiai A, Esumi H, Soga T, Tomita M. Metabolomic profiling of lung and prostate tumor tissues by capillary electrophoresis timeof-flight mass spectrometry. Metabolomics. 2013; 9:444-53.

53. Taki K, Wang B, Nakajima T, Wu J, Ono T, Uehara Y, Matsumoto $\mathrm{T}$, Oghiso $\mathrm{Y}$, Tanaka $\mathrm{K}$, Ichinohe $\mathrm{K}$, Nakamura S, Tanaka S, Magae J, et al. Microarray analysis of differentially expressed genes in the kidneys and testes of mice after long-term irradiation with low-dose-rate gammarays. J Radiat Res. 2009; 50:241-52.

54. Chou TC. Drug combination studies and their synergy quantification using the Chou-Talalay method. Cancer Res. 2010; 70:440-6. 